\title{
TOPICAL MAINTENANCE TREATMENTS IN CHRONIC DERMATITIS
}

\author{
MARIA ROTARU ${ }^{1}$, IRINA MIHAELA MATRAN ${ }^{2}$, GABRIELA MARIANA IANCU ${ }^{3}$ \\ 1,3 “Lucian Blaga” University of Sibiu, ${ }^{13}$ County Clinical Emergency Hospital, Sibiu " “George Emil Palade” University of Medicine, Pharmacy, \\ Science and Technology, Târgu Mureş
}

\begin{abstract}
Keywords: $\quad$ chronic Abstract: The epidemiological importance of chronic dermatitis is constantly growing, this pathology dermatitis, treatment, side being more and more common regardless of age, in most countries of the world. The pathogenesis of effects chronic dermatitis is complex and involves aeroallergens, immunological, dietary, climatic and psychosomatic factors. Due to the frequent recurrences but also the side effects of allopathic medicines, the number of patients with chronic dermatitis who opt for alternative treatments is increasing. In this paper we have identified allopathic topical treatments versus alternative topical treatments used in chronic dermatitis for which there is scientific evidence. The PubMed and Research Gate databases were analysed, the analysed period being 2007 - 2019. The search criteria were "chronic dermatitis", "atopic dermatitis", "psoriasis", "alternative treatments", "natural treatments", "complementary treatments"," treatments for chronic dermatitis". We also analysed the mechanisms of action of drugs or active substances used as well as side effects secondary to allopathic and alternative therapies. New treatment options in atopic dermatitis, currently under evaluation, are topical inhibitors of phosphodiesterase and Janus kinase. For patients with chronic dermatitis who want alternative therapies to allopathic ones, there are products based on oat (Rhealba variety), camphor leaves, hydroxytyrosol, enzymatically interstered fats etc.
\end{abstract}

\section{INTRODUCTION}

The most commonly known chronic inflammatory disease is atopic dermatitis (AD). This condition can progress from mild to severe forms, with risk of complications. It is most common among children (up to 25\%) and rarer among adults (2$3 \%$ ) in the majority of countries around the world, with a high prevalence in the developed countries.(1,2) Clinically, AD is characterized by erythematous-squamous lesions, intensely itchy, sometimes with exudation and the possibility of superinfection. These aspects greatly affect the quality of life of the child with $\mathrm{AD}$ but also of the parents. The $\mathrm{AD}$ etiopathogenesis is complex. The involved factors are multiple: genetic factors (filaggrin genes), skin barrier defects (3), psychosocial factors, infections, and some foods, aeroallergens, climate changes, and immunological factors.(4) The choice of allopathic or alternative therapy for the treatment of chronic dermatitis (CD) should be made after confirmation of the diagnosis and taking into account the following factors: causes of its occurrence, age, sex of patients, anamnestic details, drug treatments administered before diagnosis, informing the patient about side effects of some therapies (ex. skin atrophy after prolonged use of topical dermatocorticoids). Also, the choice of therapeutic method must be made according to the psychosomatic factors specific to each individual.

Regardless of the people, traditional medicine had its followers, more or less trained in terms of pharmacology and toxicology, respectively. Depending on the alternative therapy applied, long-term consumption of herbs can lead to a number of side effects (5) or interactions between drugs and herbs, or between herbs and foods, with adverse effects on the health of the individual.

In chronic dermatitis, there can be used topical antiinflammatory and/or immunomodulatory therapies in combination with emollients, moisturizers and special shower gels for cleansing the skin and alternative topical therapies. These will maintain the integrity of skin barrier, respectively the physiological hydro-lipid layer of the skin.(1,6-11) In the treatment of chronic dermatitis among the alternative methods for which there is scientific evidence we list: phytotherapeutic complex - PTQX (1), biological extract of Aquaphilus dolomiae (12) with anti-inflammatory and immunomodulatory action, organic oat plant extract Rhealba variety without protein, protected by three patents of international inventions (WO2010/ 054879A2, WO2010/054878 and FR2938439) (6), green tea (13), enzyme-mediated fat preparation (14), tetramethoxyluteoline (9), eupatiline (10) etc.

Currently, in chronic dermatitis, it is taken into account that during exacerbations, topical therapies have a local anti-inflammatory action through the use of dermatocorticoids, calcineurin inhibitors and glutathione synthesis derivatives. During calm periods of dermatitis, the use of emollient creams and gentle washing gels with moisturizing effect is insisted on.

Also, by analysing personal and family history, individual factors (age, type of lesions, affected body areas) and identified etiological factors, personalized therapeutic schemes can be achieved, much more effective. New promising treatment options in atopic dermatitis are topical inhibitors of phosphodiesterase and Janus kinase.

\footnotetext{
${ }^{3}$ Corresponding author: Gabriela Mariana Iancu, Str. L. Blaga, Nr. 2A, Sibiu, România, E-mail: mgabiancu@yahoo.com, Phone: +40744 372164
} Article received on 18.06.2019 and accepted for publication on 10.08.2020 


\section{CLINICAL ASPECTS}

Our paper summarizes the treatment alternatives nowadays and the new perspectives of topical therapy in $\mathrm{CD}$, with a focus on $\mathrm{AD}$.

\section{MATERIALS AND METHODS}

To identify allopathic topical treatments versus alternative topical treatments for which there is scientific evidence, we analysed the PubMed and Research Gate databases, the period analysed being 2007 - 2019. The search words were "chronic dermatitis", "atopic dermatitis", "alternative treatments", "natural treatments", "complementary treatments", "treatments for chronic dermatitis". We also analysed the mechanisms of action of these treatments

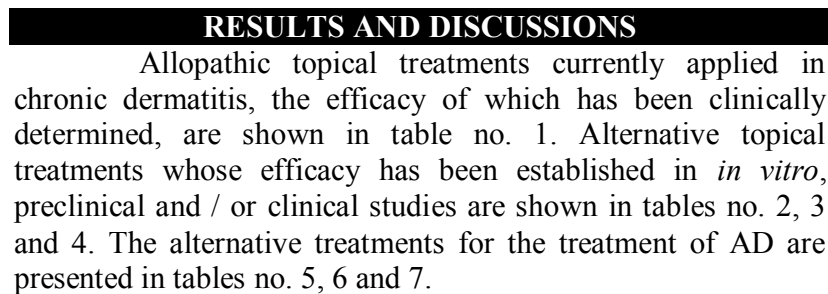

Table no. 1. Allopathic topical treatments currently applied in chronic dermatitis, the effectiveness of which has been determined clinically

\begin{tabular}{|c|c|c|}
\hline $\begin{array}{l}\text { Active substance/ } \\
\text { Drug }\end{array}$ & $\begin{array}{l}\text { The main action monitored } \\
\text { and demonstrated }\end{array}$ & Mechanism of action of the drug / active substance \\
\hline $\begin{array}{l}\text { Pimecrolimus } \\
\text { Tacrolimus simultaneously with topical steroids }(2,15)\end{array}$ & Treatment of $\mathrm{AD} *$, psoriasis & Calcineurin $\downarrow$ \\
\hline Tofacitinib $2 \%(16)$ & $\begin{array}{l}\mathrm{AD} \text { treatment, } \\
\text { Psoriasis }\end{array}$ & $\begin{array}{l}\text { - JAK } \downarrow * \text { : IL-21, IL-15, IL-13, IL-9, IL-7, IL-4, IL-2 * } \\
\text { - keratinocyte regrowth, } \\
\text { - proinflammatory factors } \downarrow \text {, } \\
\text { - antimicrobial activity. }\end{array}$ \\
\hline Crisaborol, $0.5 \%$ and $2 \%(15,17,18)$ & AD treatment & $\begin{array}{l}\text { - PDE } 4 \downarrow \text {, } \\
\text { - Adjusting the level of cAMP, } \\
\text { - Regulation of T cell activity and TH2 } * \downarrow, \text { TH17 / TH22 } \downarrow, \text { TH1 } \downarrow \text {, } \\
\text { IL-1, IL-13, IL-22, IFN- } \gamma * \text { TNF } * \downarrow .\end{array}$ \\
\hline Vitreoscilla filiformis nepatogen $5 \%$ (19) & AD treatment & $\begin{array}{l}\text { - Probiotic action } \uparrow \text {, } \\
\text { - Colonization of staphylococcus aureus of the skin } \downarrow \text {. }\end{array}$ \\
\hline Ruxolitinib (20) & AD treatment & $\begin{array}{l}- \text { PDE } 4 \downarrow \\
- \text { JAK } 1 \text { and JAK } 2 \downarrow .\end{array}$ \\
\hline $\begin{array}{l}\text { Halobetasol (21) } \\
\text { (Corticosteroid) }\end{array}$ & $\begin{array}{l}\text { AD treatment, } \\
\text { Psoriasis }\end{array}$ & $\begin{array}{l}\text {-Antiinflammatory } \uparrow: \text { PLA2 } * \downarrow, \\
\text { - Immunosuppressant } \uparrow, \\
\text { - Anti-mitogen } \uparrow \text {. }\end{array}$ \\
\hline PAC- $14028 * 0.1 \%, 0.3 \% ; 1.0 \%(22)$ & AD treatment & -Antiinflammatory $\uparrow:$ TRPV1 $* \downarrow($ PLA2 $\downarrow$, LOX $12 * \downarrow)$. \\
\hline LPSp cream * $(23)$ & $\mathrm{AD}$ treatment & $\begin{array}{l}\text { - Interaction with cells in the stratum corneum of the epidermis, } \\
\text { - Filaggrin } \uparrow \text {, } \\
\text { - } \beta \text {-defensin } \uparrow \text { (antimicrobial action), } \\
\text { - TH } 2 \downarrow \text {. }\end{array}$ \\
\hline
\end{tabular}

*AD - atopic dermatitis; HL: Hodgkin's lymphoma; B-cell or B-cell NHL: B-cell/T-cell non-Hodgkin's lymphoma; JAK - Janus kinase; IL - interleukin; PDE4 Phosphodiesterase E4; cAMP - ciclic adenozine monofosfat; TH - helper T cells; TNF - tumor necrosis factor; IFN - interferon; PLA2 - Phospholipase A2; PAC-14028 TRPV1 Antagonist (Transient Receptor Potential Vaniloid Subfamily, Member 1 (TRPV1)); LOX 12 - Lipoxygenase 12; TRPV - transient receptor potential cation channels vanilloid; LPSp - lipopolysaccharide derived from Pseudomonas Agglomerans.

Table no. 2. Alternative topical treatments currently applied in chronic dermatitis, the efficacy of which has been determined in vitro

\begin{tabular}{|c|c|c|}
\hline Active substance & The main action monitored and demonstrated & Mechanism of action of the drug / active substance \\
\hline $\begin{array}{l}\text { Fenolia }{ }^{\circledR} \text { Eudermal } \\
\text { Cream } 15 \text { (HT * } \\
\text { formulation) (8) }\end{array}$ & $\begin{array}{l}\text { - RHE thickness *, } \\
\text { - Adequate maturation and protein expression, } \\
\text { - Antiinflammatory action, } \\
\text { - HT availability. }\end{array}$ & $\begin{array}{l}\text { - RHE thickness: Cell proliferation (Ki67 } * \text { ) } \uparrow \text {, } \\
\text { - Adequate maturation and protein expression (Loricrin, Fillagrin, E-Cadherin and } \\
\text { Cytokeratine } 5 \text { and } 6 \uparrow \text { ), } \\
\text { - Antiinflammatory (IL } 1 * \downarrow \text {, IL } 8 \downarrow \text { ), } \\
\text { - Transcutaneous absorption for HT } \uparrow \text {. }\end{array}$ \\
\hline
\end{tabular}

Table no. 3. Alternative topical treatments currently applied in chronic dermatitis, the efficacy of which has been preclinically determined

\begin{tabular}{|c|c|c|c|c|c|c|c|}
\hline \multirow{2}{*}{$\begin{array}{l}\text { The product/ } \\
\text { preparation, } \\
\text { dose, method of } \\
\text { administration }\end{array}$} & \multicolumn{7}{|c|}{ Preclinical determinations of efficacy of products / preparations } \\
\hline & $\begin{array}{l}\text { Pathology } \\
\text { followed }\end{array}$ & $\begin{array}{l}\text { Species / Line, } \\
\text { sex, age } \\
\text { (Seven weeks) }\end{array}$ & $\begin{array}{l}\text { No. of } \\
\text { Animals / } \\
\text { No. of } \\
\text { Groups }\end{array}$ & $\begin{array}{c}\text { Period } \\
\text { (number of } \\
\text { days) }\end{array}$ & $\begin{array}{l}\text { The mechanism of } \\
\text { pathology } \\
\text { induction }\end{array}$ & $\begin{array}{l}\text { The main action } \\
\text { monitored and } \\
\text { demonstrated }\end{array}$ & $\begin{array}{c}\text { Product / Mechanism of } \\
\text { Action } \\
\text { preparation }\end{array}$ \\
\hline $\begin{array}{l}\text { Eupatiline } 1 \% * \\
(10)\end{array}$ & $\mathrm{AD}^{*}$ & $\begin{array}{l}\text { Lab rats } \\
\mathrm{NC} / \mathrm{Nga} \text { male, } \\
4\end{array}$ & $\begin{array}{l}5 \text { animals / } \\
\text { group; } \\
4 \text { groups }\end{array}$ & 29 & DNCB* & $\begin{array}{l}\text { Clinical severity } \\
\text { score for } \mathrm{AD}\end{array}$ & $\begin{array}{l}\text { - T-SLP *, TNF- } \alpha \text { *, Th2 *, } \\
\text { IL-4 *, IL-19 } \downarrow \text {, } \\
\text { - Hyperkeratosis } \downarrow .\end{array}$ \\
\hline
\end{tabular}

*AD - atopic dermatitis; Eupatiline - 2 - [3,4-dimethoxyphenyl]-5,7-dihydroxy-6-methoxychromen-4-one (a lipophilic flavonoid obtained from medicinal plants of Artemisia umbelliformis Lam. and Artemisia genipi Weber); DNCB-1-chloro-2,4-dinitrobenzene; T-SLP - thymic stromal lymphopoietin; TNF - tumor necrosis factor; Th - helper T cells; IL - interleukin.

Table no. 4. Alternative treatments for the treatment of chronic dermatitis, the efficacy of which has been determined preclinically

\begin{tabular}{|c|c|c|c|c|c|c|c|}
\hline \multirow{2}{*}{$\begin{array}{l}\text { The product/ } \\
\text { preparation, } \\
\text { dose, method of } \\
\text { administration }\end{array}$} & \multicolumn{7}{|c|}{ Preclinical determinations of efficacy of products / preparations } \\
\hline & $\begin{array}{l}\text { Pathology } \\
\text { followed }\end{array}$ & $\begin{array}{l}\text { Species / } \\
\text { Line, sex, } \\
\text { age } \\
\text { (Weeks) }\end{array}$ & $\begin{array}{c}\text { No. of } \\
\text { Animals / Nr. } \\
\text { of Groups }\end{array}$ & $\begin{array}{l}\text { Period, } \\
\text { no. of } \\
\text { days }\end{array}$ & $\begin{array}{l}\text { The mechanism } \\
\text { of pathology induction }\end{array}$ & $\begin{array}{l}\text { The main action } \\
\text { monitored and } \\
\text { demonstrated }\end{array}$ & $\begin{array}{l}\text { Mechanism of } \\
\text { action a } \\
\text { product / } \\
\text { preparation }\end{array}$ \\
\hline $\begin{array}{l}\text { PTQX (1), } \\
8.0 \mathrm{~g} / \mathrm{kg} \mathrm{c} \text {, } \\
\text { dissolved in water, } \\
20 \mathrm{ml} / \mathrm{kg}\end{array}$ & $\mathrm{AD}^{*}$ & $\begin{array}{l}\text { Lab rats } \\
\mathrm{NC} \mathrm{/} \mathrm{Nga,} \\
\text { male, } \\
6-8 \text { weeks }\end{array}$ & $\begin{array}{l}7-8 \text { animals / } \\
\text { group; } \\
4 \text { groups }\end{array}$ & 21 & $\begin{array}{l}\text { - DNCB determines } \downarrow \\
\text { release of endogenous and } \\
\text { exogenous histamine *, } \\
\text { - Inflammatoryeffects: }\end{array}$ & $\begin{array}{l}\text { - Inflammation of } \\
\text { CD4 }+* \text { and CD8 T } \\
\text { cells in skin lesions } \downarrow \text {, } \\
\text { - IgE production * in }\end{array}$ & $\begin{array}{ll}\text { Mast } & \text { cell } \\
\text { infiltration } & \text { into } \\
\text { skin lesions } \downarrow & \end{array}$ \\
\hline
\end{tabular}




\section{CLINICAL ASPECTS}

\begin{tabular}{|c|c|c|c|c|c|c|c|}
\hline & & & & & $\begin{array}{l}\text { > edema with xylene } \\
\text { inserted into the ear, } \\
>\text { inflammation with } \\
\text { acetic acid in the } \\
\text { permeable capillaries of } \\
\text { the abdomen. }\end{array}$ & $\begin{array}{l}\text { serum } \downarrow \text {, } \\
\text { - Swelling of the ear } \downarrow \text {, } \\
\text { - Adjusting the balance } \\
\text { of Th / Treg cells * }\end{array}$ & \\
\hline & & $\begin{array}{l}\text { Lab rats } \\
\text { C57BL / } 6\end{array}$ & $\begin{array}{l}7-8 \text { animals } / \\
\text { group; } \\
4 \text { groups }\end{array}$ & 21 & $\begin{array}{l}\text { Differentiation of Th1 and } \\
\text { Th2 cells }\end{array}$ & $\begin{array}{l}\text { Balance adjustment } \\
\text { between Th1 and Th2 }\end{array}$ & \\
\hline $\begin{array}{l}\text { Balance } \\
\text { adjustment } \\
\text { between Th1 and } \\
\text { Th2 }\end{array}$ & $\begin{array}{l}\mathrm{AD}, \\
\text { Psoriasis }\end{array}$ & $\begin{array}{l}\text { BALB / c } \\
\text { females } \\
7 \text { weeks } \\
\text { us }\end{array}$ & $\begin{array}{l}8 \quad \text { animals } \\
\text { group } \\
4 \text { groups }\end{array}$ & 31 & DNCB application & $\begin{array}{l}\text { - MDC } * \downarrow \\
\text { - phosphorylated } \\
\text { STAT1* (tyrosine } 701 \\
\text { and serine } 727 \text { ), } \\
\text { - Phosphorylation ERK } \\
1 / 2 * \downarrow, \\
\text { - IgE } * \downarrow .\end{array}$ & $\begin{array}{l}\text { Action on skin } \\
\text { keratinocytes }\end{array}$ \\
\hline Vinegar (11) & $\mathrm{AD}$ & $\begin{array}{l}\text { Lab rats } \\
\text { Murinae, } \\
\text { females, } \\
\text { NS * }\end{array}$ & $\begin{array}{l}6 \quad \text { animals } \\
\text { group } \\
5 \text { groups }\end{array}$ & 21 & $\begin{array}{l}\text { Topical application } 0.1 \% \\
\text { Ox * }\end{array}$ & $\begin{array}{l}\text { - Edema, scales, } \\
\text { lichenification } \downarrow \text {, } \\
\text { - Epidermal } \\
\text { permeability } \uparrow \text {, } \\
\text { - Skin hydration } \uparrow\end{array}$ & $\begin{array}{l}\text { Changing the } \mathrm{pH} \\
\text { of the skin }\end{array}$ \\
\hline
\end{tabular}

*AD - atopic dermatitis; PTQX - TCM Pei Tu Qing (phytotherapeutic preparation from traditional Chinese medicine recommended in the treatment of AD); DNCB-1 chloro-2,4-dinitrobenzene; IgE - Immunoglobulins, CD - cluster of differentiation; Th - helper T cells; Treg - regulatory T cells; MDC - inflammatory chemokines; STAT 1 - Signal translator and transcription activator 1; ERK $1 / 2$ - Kinases regulated by extracellular signal (synonym for mitogenated protein kinase (MAPK)); Ig E immunoglobulins E; NS - Not specified; Ox - Oxazolone.

Table no. 5. Alternative topical treatments currently applied in chronic dermatitis, the effectiveness of which has been determined clinically

\begin{tabular}{|c|c|c|c|}
\hline Active substance & $\begin{array}{c}\text { The main action monitored and } \\
\text { demonstrated }\end{array}$ & Mechanism of action of the drug / active substance & Side effects \\
\hline $\begin{array}{l}\text { Enzymatically intersteratted } \\
\text { fats (14) }\end{array}$ & AD treatment * & $\begin{array}{l}\text { - Absorption into the skin } \uparrow \text {, } \\
\text { - Oily deposits on the skin } \downarrow \text {, } \\
\text { - Skin hydration } \uparrow \text {. }\end{array}$ & They were not reported \\
\hline 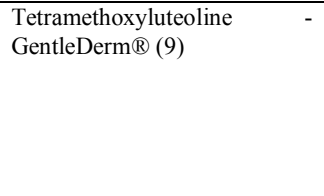 & $\begin{array}{l}\text { AD treatment, } \\
\text { psoriasis, } \\
\text { mastocytosis }\end{array}$ & $\begin{array}{l}\text { - MC * } \downarrow \text {, } \\
\text { - Antioxidant } \uparrow, \\
\text { - Itching, } \\
\text { - Keratinocytes } \uparrow, \\
\text { - Histamine } \downarrow \text {, } \\
\text { - IL-6, IL-8 } *, \text { TNF } \alpha * \downarrow \text {, } \\
\text {-Tryptase from MCs } * \downarrow .\end{array}$ & They were not reported \\
\hline
\end{tabular}

* AD - atopic dermatitis; MC - cutaneous mastocytosis; IL - interleukins; TNF $\alpha$ - tumor necrosis factor; MCs - neoplastic mast cells.

Table no. 6. Alternative treatments currently applied in chronic dermatitis, the efficacy of which has been determined in vitro

\begin{tabular}{|c|c|c|}
\hline Active substance & The main action monitored and demonstrated & Mechanism of action of the drug / active substance \\
\hline Organic oat plant extract variety Rhealba (6) & $\mathrm{AD}$ treatment $*$ & $\begin{array}{l}\text {-PGI2 (PG6K) } * \downarrow ; \\
\text {-Th1 and Th2 } * \downarrow ; \\
\text { - IL-2, IL-4, IL-13* } \downarrow \text {; }\end{array}$ \\
\hline $\begin{array}{l}\text { Organic oat plant extract variety Rhealba - } \\
\text { topic (6) }\end{array}$ & AD treatment & $\begin{array}{l}\text {-TSLP } * \downarrow ; \\
\text {-Keratinocyte } \uparrow ; \\
\text {-PLA2 and COX-2 } * \downarrow ;\end{array}$ \\
\hline
\end{tabular}

*AD - atopic dermatitis; PGI2 (PG6K) - prostaglandins I2, 6K; TH - Helper T lymphocytes (Th cells); IL - interleukin; PLA2 - Phospholipase A2; COX Cyclooxygenase-2; TSLP - Thymic stromal lymphopoietin.

Table no. 7. Alternative treatments currently applied in chronic dermatitis, the effectiveness of which has been determined clinically

\begin{tabular}{|c|c|c|c|}
\hline Active substance & $\begin{array}{l}\text { The main action } \\
\text { monitored and } \\
\text { demonstrated }\end{array}$ & Mechanism of action of the drug / active substance & Side effects \\
\hline $\begin{array}{l}\text { Organic oat plant extract } \\
\text { variety Rhealba (6) }\end{array}$ & $\mathrm{AD}$ treatment & $\begin{array}{l}\text { - Repair of the epidermal barrier: } \\
\text { > Keratinocyte differentiation; } \\
\text { > Filaggrin } \uparrow ; \\
\text { > Epidermal lipids (ceramides, cholesterol and fatty acids) } \uparrow ; \\
\text { > Anti-inflammatory activity } \uparrow(\text { PLA } * \downarrow, \text { COX- } 2 * \downarrow) ; \\
>\text { Immunomodulatory activity } \uparrow(\text { PGI- } 2 * \downarrow, \text { IL- } 2 *), \text { MHC-II } * \downarrow, \text { TH2 } \\
* \text {, IL-13 and IL-4 } \downarrow\end{array}$ & They were not reported \\
\hline
\end{tabular}

*AD - atopic dermatitis; PLA2 - Phospholipase A2; COX - Cyclooxygenase-2; PGI-2 - Prostaglandin I2; IL - interleukin; MHC-II - class of major complex histocompatibility molecules; TH - Helper T lymphocytes (Th cells).

For patients suffering from $\mathrm{AD}$ and wanting alternative treatments instead of allopathic ones, there are products based on PTQX (1), organic oat plant extract variety Rhealba (6), organic oat plant extract variety Rhealba - topic (6), camphor tree (Cinnamomum camphora) - leaves, $80 \%$ alcoholic extract (7), Fenolia ${ }^{\circledR}$ Eudermal Cream 15 (hydroxytyrosolbased formulas) [8], enzymatically interspersed fats (14), tetramethoxyluteoline - GentleDerm $\AA(9)$, Eupatiline 1\%*(10), vinegar.(11) In the case of products with natural extracts, flavonoids have an anti-inflammatory role with positive effects in the treatment of DA. The strong anti-inflammatory (PLA2
COX $-2 \downarrow$ ) effect of the organic oat plant extract variety Rhealba (6) is determined by flavonoids (polyphenols) and saponins (polar molecules). And in the case of GentleDerm ${ }^{\circledR}$ (9), flavonoids have anti-inflammatory action, tetramethoxyluteoline being a natural flavonoid with antioxidant properties. Another flavonoid that enhances DA is eupatilin (5,7-dihydroxy-30,40,6trimethoxyflavone).(10)

In clinical practice, the treatment of $\mathrm{AD}$ in some cases can be quite difficult, both due to frequent recurrences and the use of various cosmetics with perfumes (detergents, balms, soaps etc) 


\section{CONCLUSIONS}

An important role belongs to the dermatologist in the control of the acute phase of $\mathrm{AD}$ but also later in the maintenance of atopic skin with special care products with calming, emollient and moisturizing effects, which do not destroy the hydro-lipid barrier and thus affected in AD.

\section{REFERENCES}

1. Yan F, Zhang J, Li X, Mo X, Liu J, Ye S, et al Therapeutic Effects of Chinese Herbal Formula (PTQX) on NC/Nga Mice with Atopic Dermatitis-Like Skin Lesions. Evid Based Complement Alternat Med. 2019;2019:1-13.

2. Arellano FM, Wentworth CE, Arana A, Fernández C, Paul $\mathrm{CF}$. Risk of lymphoma following exposure to calcineurin inhibitors and topical steroids in patients with atopic dermatitis. J Invest Dermatol. 2007;127(4):808-816.

3. Rotaru M, Ionescu A, Rotaru BI, Iancu GM. Etiopathogenic and therapeutic considerations in atopic dermatitis. Acta Medica Transilvanica. 2019;24(2):34-38.

4. Mitchell AE. Bidirectional relationships between psychological health and dermatological conditions in children. Psychol Res Behav Manag. 2018;11:289-298.

5. Sadr Mohammadi R, Bidaki R, Mirdrikvand F, Mostafavi Yazdi SN, Yazdian Anari P. Peganum Harmala (Aspand) Intoxication; a Case Report. Emerg (Tehran). 2016;4(2):106-107.

6. Wollenberg A, Folster-Holst R, Saint Aroman M et al. Effects of a protein-free oat plantlet extract on microinflammation and skin barrier function in atopic dermatitis patients. JEADV. 2018;32 (Suppl. 1):1-15.

7. Kang NJ, Han SC, Yoon SH, Yoon JY, Maeng YH, Kang $\mathrm{HK}$, et al. Cinnamomum camphora Leaves Alleviate Allergic Skin Inflammatory Responses In Vitro and In Vivo. Toxicol Res. 2019;35(3):279-285.

8. Smeriglio A, Denaro M, Mastracci L, Grillo F, Cornara L, Shirooie S, et al. Safety and efficacy of hydroxytyrosolbased formulation on skin inflammation: in vitro evaluation on reconstructed human epidermis model. Daru. 2019;27(1):283-293.

9. Theoharides TC, Stewart JM, Tsilioni I. Tolerability and benefit of a tetramethoxyluteolin-containing skin lotion. Int J Immunopathol Pharmacol. 2017;30(2):146-151.

10. Lee JH, Lee YJ, Lee JY, Park YM. Topical Application of Eupatilin Ameliorates Atopic Dermatitis-Like Skin Lesions in NC/Nga Mice. Ann Dermatol. 2017;29(1):6168.

11. Lee NR, Lee HJ, Yoon NY, Kim D, Jung M, Choi EH. Application of Topical Acids Improves Atopic Dermatitis in Murine Model by Enhancement of Skin Barrier Functions Regardless of the Origin of Acids. Ann Dermatol. 2016;28(6):690-696.

12. Aries MF, Hernandez-Pigeon $\mathrm{H}$, Vaissière $\mathrm{C}$, Delga $\mathrm{H}$, Caruana A, Lévêque $\mathrm{M}$, et al. Anti-inflammatory and immunomodulatory effects of Aquaphilus dolomiae extract on in vitro models. Clin Cosmet Investig Dermatol. 2016;9:421-434.

13. Rashmi S, Nahid A, Tariq M. H. Green tea polyphenol epigallocatechin-3-gallate: inflammation and arthritis. Life Sci. 2010;86(25-26):907-918.

14. Kowalska M, Mendrycka M, Zbikowska A, Kowalska D. Assessment of a stable cosmetic preparation based on enzymatic intersterified fat, proposed in the prevention of atopic dermatitis. Acta Pol Pharm. 2017;74(2):465-476.

15. Stein Gold LF, Spelman L, Spellman MC, Hughes MH, Zane LT. A Phase 2, Randomized, Controlled, Dose-
Ranging Study Evaluating Crisaborole Topical Ointment, $0.5 \%$ and $2 \%$ in Adolescents With Mild to Moderate Atopic Dermatitis. JDD. 2015;14(12):1394-1399

16. Bissonnette R, Papp KA, Poulin Y, Gooderham M, Raman $\mathrm{N}$, Mallbris L, et al. Topical tofacitinib for atopic dermatitis: a phase IIa randomized trial. Br J Dermatol. 2016;175(5):902-911.

17. Bissonnette R, Pavel AB, Diaz A, Werth JL, Zang C, Vranic I, et al. Crisaborole and atopic dermatitis skin biomarkers: An intrapatient randomized trial. J Allergy Clin Immunol. 2019;144:1274-89.

18. Paller AS, Tom WL, Lebwohl MG, Blumenthal RL, Boguniewicz M, Call RS, et al. Efficacy and safety of crisaborole ointment, a novel, nonsteroidal phosphodiesterase 4 (PDE4) inhibitor for the topical treatment of atopic dermatitis (AD) in children and adults. J Am Acad Dermatol. 2016;75(3):494-503.

19. Gueniche A, Knaudt B, Schuck E et al. Effects of nonpathogenic gram-negative bacterium Vitreoscilla filiformis lysate on atopic dermatitis: a prospective, randomized, double-blind, placebo-controlled clinical study. Br J Dermatol. 2008;159:1357-1363.

20. Kim BS, Howell MD, Sun K, Papp K, Nasir A, Kuligowski ME, et al. Treatment of atopic dermatitis with ruxolitinib cream (JAK1/JAK2 inhibitor) or triamcinolone cream. J Allergy Clin Immunol. 2019;1-12.

21. Awad N, Preuss CV. Halobetasol Cream. [Updated 2020 Feb 20]. In: StatPearls [Internet]. Treasure Island (FL): StatPearls Publishing; 2020 Jan. Available from:https://www.ncbi.nlm.nih.gov/books/NBK544234/

22. Lee YW, Won CH, Jung K, Nam HJ, Choi G, Park YH, et al. Efficacy and safety of PAC-14028 cream - a novel, topical, nonsteroidal, selective TRPV1 antagonist in patients with mild-to-moderate atopic dermatitis: a phase IIb randomized trial. Br J Dermatol. 2019;180(5):10301038.

23. Nakai K, Kubota Y, Soma GI, Kohchi C. The Effect of Lipopolysaccharide-containing Moisturizing Cream on Skin Care in Patients With Mild Atopic Dermatitis. In Vivo. 2019;33(1):109-114. 